\title{
Organización y funcionamiento de los agrupamientos en Educación Superior: concepciones de los estudiantes
}

\author{
Organization and performance of students' grouping in Higher Education: students' \\ conceptions
}

\author{
Ana Torres Soto (iD \\ ana.t.s@um.es \\ Universidad de Murcia. España \\ Mónica Vallejo Ruíz (iD \\ monicavr@um.es \\ Universidad de Murcia. España
}

\section{Resumen}

Tanto el uso apropiado de metodologías activas en los procesos de enseñanza como el incremento de la participación y responsabilidad compartida del alumnado de su propio aprendizaje siguen siendo retos del sistema educativo. Para avanzar en este último sentido, crear espacios verdaderamente cooperativos de aprendizaje es un factor estratégico clave. Este trabajo pretende contribuir al conocimiento de la influencia de criterios, normas y funcionamiento de los agrupamientos en el aprendizaje cooperativo en Educación Superior. Específicamente, la finalidad del presente estudio es conocer las valoraciones de los estudiantes sobre la organización y el funcionamiento de los grupos de trabajo más idóneos para alcanzar un aprendizaje realmente cooperativo. El estudio se ha focalizado en asignaturas en las que se ha empleado la estrategia metodológica Aprendizaje Basado en Problemas -ABP, en adelante- en dos universidades de la Región de Murcia. Se ha aplicado la escala ACOES a 75 estudiantes de primer curso del grado de Educación Infantil de la Universidad de Murcia y de ISEN, Centro Universitario. Los resultados muestran una correspondencia entre las valoraciones de los estudiantes sobre el funcionamiento de los agrupamientos y las condiciones que en la literatura se han planteado como idóneas para que se desarrolle un aprendizaje cooperativo auténtico. Entre esas valoraciones se destaca la necesidad de que se expliciten normas en el grupo, que las agrupaciones sean realizadas a criterio de los estudiantes y que el trabajo cooperativo esté altamente estructurado y controlado por el docente.

Palabras clave: aprendizaje cooperativo; agrupamientos; competencias; educación superior; colaboración.

\begin{abstract}
Both the appropriate use of active methodologies in teaching processes and the increased participation and shared responsibility of students for their own learning remain challenges of the education system. To make progress in this last sense, creating truly cooperative learning spaces is a key strategic factor. This paper aims to contribute to the knowledge of the influence of criteria, norms and functioning of groupings in cooperative learning in Higher Education. Specifically, the purpose of the present study is to know the students' evaluations about the organization and functioning of the working groups best suited to achieve truly cooperative learning. The study has focused on subjects in which the methodological strategy based on Problem-Based Learning (ABP) has been used in two universities in the Region of Murcia. The ACOES scale has been applied to 75 first-year undergraduate students at the University of Murcia and ISEN. The results indicate a correlation between the students' assessments of the functioning of the groupings and the conditions that in the literature have been considered as suitable for the development of authentic cooperative learning. Among these assessments is the need for rules to be made explicit in the group, for groups to be made at the discretion of the students and for cooperative work to be highly structured and controlled by the teacher.
\end{abstract}

Keywords: cooperative learning; students' grouping; competences; higher education; collaboration.

Cómo referenciar este artículo / How to reference this article:

Torres, A., \& Vallejo, M. (2018). Organización y funcionamiento de los agrupamientos en Educación Superior: concepciones de los estudiantes. Tendencias Pedagógicas, 32, 15-30.doi: 10.15366/tp2018.32.002 


\section{Introducción}

El trabajo en grupo se configura como uno de los elementos clave de los currículos universitarios tras el proceso de integración de las universidades en el Espacio Europeo de Educación Superior (en adelante EEES). Se asume como una competencia general para el desarrollo de una Educación Superior de calidad, junto con el logro de otras competencias generales, ligadas todas ellas a las demandas exigidas por la sociedad y el mercado laboral actual (Michavila, Martínez, MartínGonzález, García, y Cruz-Benito, 2016). A su vez, se entiende el trabajo en grupo no solo como una competencia transversal que debiera incorporarse a los programas formativos, sino como una metodología de aprendizaje centrada en el estudiante que, de acuerdo con Atxurra, VillardónGallego y Calvete (2015), si es aplicada eficazmente, llevará al desarrollo de otras competencias útiles para la vida social y profesional.

El aprendizaje cooperativo, que implica el trabajo en grupo, se torna como una estrategia organizativa alternativa y complementaria a las técnicas tradicionales, que sirve para organizar los procesos cognitivos en el aprendizaje tanto fuera como dentro del aula (González y García, 2007). Entre los principales precursores del aprendizaje cooperativo se encuentran Johnson, Johnson y Holubec (1999) y Kagan (1999), quienes señalaron que la cooperación supone, entre otros aspectos, trabajar juntos para alcanzar objetivos comunes y obtener resultados que sean beneficiosos para el propio alumno y para todos los demás miembros del grupo. Así, "una situación cooperativa con éxito estará supeditada al éxito del grupo" (Hancock, 2004, 160).

Tomando como base estas y otras aportaciones sobre el aprendizaje cooperativo, el escenario educativo universitario aconseja impulsar entornos y actividades interactivas donde los estudiantes puedan trabajar en grupo y aprender de manera cooperativa. Pero trabajar en grupo no implica necesariamente cooperar; se han de diseñar y estructurar una serie de condiciones para que la situación de enseñanza y aprendizaje sea realmente cooperativa (Lata y Castro, 2016), y esto va a depender del "conjunto de elementos y de operaciones que se suceden en el desarrollo de la actividad" (Pujolás, 2009, 228). En este trabajo se trata de vislumbrar aquellos aspectos estructurales y organizativos que interfieren en la organización de los grupos y, consecuentemente, en el aprendizaje cooperativo. Para ello, se presentan los resultados de una investigación que se ha realizado en el marco de dos asignaturas en las cuales se ha trabajado con la metodología ABP, utilizando el aprendizaje cooperativo como estrategia metodológica para su funcionamiento.

\subsection{Importancia de aprender a cooperar en la universidad: traslación a las instituciones escolares}

En la formación de estudiantes universitarios, especialmente de futuros docentes, la necesidad de conocer, comprender y poner en práctica el aprendizaje cooperativo es ineludible. Se considera una competencia esencial que posibilita el aprendizaje de habilidades y destrezas básicas para un ejercicio profesional posterior. Así, su aprendizaje y traslación al ámbito profesional (en este caso, la escuela), puede suponer la mejora de las interacciones efectivas entre el alumnado, entre los propios docentes y con la comunidad educativa (Darling-Hammond, 2008). Sin embargo, la creación de espacios verdaderamente cooperativos en las instituciones escolares es todavía un reto difícil de abordar.

El Real Decreto 1027/2011, de 15 de julio, por el que se establece el Marco Español de Cualificaciones para la Educación Superior y las sucesivas normativas que han regulado la Ordenación de las Enseñanzas Universitarias (RD. 1393/2007, RD. 861/2010, RD. 96/2014, RD. 43/2015) han subrayado que es la institución universitaria la que, incorporada a los planteamientos de un espacio común europeo, tiene la responsabilidad de potenciar en los estudiantes competencias que van más allá de saberes propios de un ámbito disciplinar (competencias específicas), deteniéndose también en el desarrollo de competencias generales (denominadas transversales hasta que fueran modificadas por requerimiento de ANECA, 2016), estrechamente ligadas a una dimensión más personal, social y laboral de las personas (Martínez y González, 2018). El hecho de que los estudiantes aprendan a trabajar de manera cooperativa en la universidad supone un valor añadido a su formación, imprescindible para establecer futuras relaciones 
profesionales constructivas y para alcanzar propósitos comunes en su profesión. En este último aspecto, numerosos son los autores (Escudero, 2009; Lledó y Perandones, 2011; Rodríguez, 2012; Bolívar, 2013) que pusieron de relieve la importancia de crear en las escuelas redes y comunidades de aprendizaje entre profesionales, entendidas como estrategia clave para el buen funcionamiento de las instituciones escolares.

A tal fin, se entiende que alcanzar la cooperación en la escuela comienza por aprender a cooperar en la universidad. Este aprendizaje ha de ser consolidado en base a las propias experiencias formativas y didácticas de los estudiantes (Fombona, Iglesias y Lozano, 2016). Como bien indica Miró (2010, p.2), "los problemas que nos encontramos en el aula (miembros que "pasan" del equipo, que no tienen su parte hecha a tiempo, que intentan aprovecharse de los demás) también existen en los equipos profesionales". Es por ello que favorecer situaciones cooperativas en las aulas universitarias, similares a las que se pueden encontrar en un futuro en el contexto escolar, son entendidas como la base para su traslación futura a las escuelas.

\subsection{Condicionantes de un aprendizaje cooperativo}

Las virtualidades del trabajo cooperativo han sido ampliamente estudiadas, señalando los efectos positivos que conllevan en contraposición a otras estructuras de actividad, como la individualista o la competitiva (Johnson y Johnson, 1987; Colomina y Onrubia, 2004; Serrano, Pons y GonzálezHerrero, 2007; Pérez, 2008; Pujolás, 2009). Entre las revelaciones que arrojan dichos estudios encontramos potencialidades y beneficios tales como la mejora del rendimiento académico, de la autoestima, actitudes positivas hacia el aprendizaje y hacia las relaciones interpersonales, favoreciendo todo ello la integración del alumnado y la consecución de estrategias de actuación democráticas.

A pesar de conocer sus beneficios, no es sencillo plantear situaciones de aprendizaje realmente cooperativas. La organización de situaciones de enseñanza y aprendizaje basadas en el aprendizaje cooperativo implican y requieren de una profunda redefinición y reestructuración de las acciones formativas y de la práctica docente. Se decía anteriormente que el aprendizaje cooperativo va más allá del trabajo en grupo. Si el trabajo en grupo implica que los estudiantes interactúen, el trabajo cooperativo añade una connotación a esa interacción que no se ve reducida al hecho de agrupar a los estudiantes, como ocurre con el trabajo en grupo (Atxurra, Villardón-Gallego y Calvete, 2015). De igual modo, el trabajo colaborativo y el trabajo cooperativo adquieren acepciones diferentes, pues mientras el primero no requiere de una estructuración en las formas de interacción, el aprendizaje cooperativo tiene que integrar, necesariamente, formas de interacción estructuradas y bien definidas (Goikoetxea y Pascual, 2002; Gómez y Hernándo, 2016). Aprender cooperativamente supone reconocer y poner en práctica una serie de condiciones básicas en esas formas de interacción y en el modo de realizar los agrupamientos.

Por un lado, refiriéndonos a las formas de interacción, autores como del Barco, Castaño, Iglesias y Marugán (2014) describen como indispensables la responsabilidad e interdependencia de los miembros del equipo para aprender lo que el profesor enseña y contribuir a que lo aprendan también sus compañeros; por su parte, Pujolás (2008) añade a esa condición la doble finalidad de utilizar el aprendizaje cooperativo tanto para aprender contenidos escolares como para aprender a trabajar en equipo, como un contenido más. Y para ello, resalta la importancia de crear equipos de trabajo estables en el tiempo. Así pues, entendemos que las interacciones que se realizan dentro del equipo en las actividades cooperativas han de llevar a los estudiantes a ayudarse de manera mutua unos a otros durante el desarrollo de las mismas; deben incorporar lo que este autor denomina "plus de solidaridad".

Estas precisiones de lo que supone la interacción en el aprendizaje cooperativo ya fueron apuntadas por diversos autores que han estudiado esta temática (Kagan, 1999; Johnson, Johnson y Holubec, 1999; Slavin, 1999; García, Traver y Candela, 2001; Prieto, 2007), por lo que se vislumbra una consideración común y compartida de lo que requiere, necesariamente, una estructura de actividad cooperativa. Tomando como base las aportaciones de Pujolás (2009), quien asimismo 
asume las contribuciones de Johnson, Johnson y Holubec (1999) y Kagan (1999), podemos definir el aprendizaje cooperativo como:

\begin{abstract}
"El uso didáctico de equipos reducidos de alumnos, generalmente de composición heterogénea en rendimiento y capacidad, aunque ocasionalmente pueden ser más homogéneos, utilizando una estructura de la actividad tal que asegure al máximo la participación igualitaria (para que todos los miembros del equipo tengan las mismas oportunidades de participación) y se potencie al máximo la interacción simultánea entre ellos, con la finalidad de que todos los miembros de un equipo aprendan los contenidos escolares, cada una hasta el máximo de sus posibilidades y aprendan, además, a trabajar en equipo" (Kagan, 1999, 231).
\end{abstract}

De acuerdo con este autor, Johnson, Johnson y Holubec (1999), en su modelo "Aprender Juntos", consideraban dos elementos o principios básicos en una estructura cooperativa: la interdependencia positiva (percepción de que no se puede lograr el objetivo de grupo si no lo logran todos y cada uno de los componentes) y la responsabilidad individual (cada miembro del grupo debe asumir su tarea individual sin olvidarse de las tareas del grupo). Sin embargo, Kagan (1999), añadía dos nuevos elementos que venían a concretar y matizar los dos anteriores, y que definía como participación igualitaria (garantizar la participación equitativa de todos los miembros del grupo, mediantes estrategias de participación igualitaria) e interacción simultánea (asegurar la interacción de todos los miembros del grupo antes de proceder a la realización de la actividad, con mecanismos/técnicas altamente estructuradas). Es crucial, por tanto, que el diseño de situaciones de aprendizaje cooperativas incorpore estos principios básicos para asegurar unas condiciones óptimas en la interacción de los estudiantes. Un paso más profundo se ha dado en un reciente estudio abordado por Jourand, Adé, Sève, Komar y Thouvarecq (2017), en el que se ha descrito y caracterizado los tipos de interacciones de los estudiantes en una materia concreta ofreciendo propuestas de intervenciones didácticas para los maestros.

Pero además de considerar las condiciones en las que se debe de dar la cooperación, es inexorable conocer qué dimensiones es necesario valorar y tener en cuenta para la organización de los agrupamientos. La planificación, constitución y funcionamiento de los grupos de trabajo cooperativos son un indicador y un condicionante, en cierto modo, de las interacciones que se producen en ellos. Ejemplo de ello son: la heterogeneidad de los grupos de trabajo (habilidades, intereses, género, etnia, etc.); la planificación y estructuración de las actividades de aprendizaje por parte de los docentes; y las normas que rigen su funcionamiento interno.

Respecto a ello, algunos autores como Ovejero (1990), Lobato (1998) o García, Traver y Candela (2001) señalan que la configuración y composición de los grupos han de ser lo más heterogéneos posibles y deben agrupar entre tres y cinco estudiantes (teniendo en cuenta las características de la actividad), de manera que se garanticen las posibilidades de intervención e interacción de todos los participantes. Así mismo, consideran que debe realizarse una distribución de roles y funciones con la intención de repartirse responsabilidades y tomar conciencia de ellas, relativas éstas a la organización del trabajo y las relaciones que se establecen en el grupo. Respecto a esta configuración y composición de los grupos se considera que han de ser actuaciones que debería de acometer el profesorado (Colomina y Onrubia, 2004), así como la definición y concreción de las tareas que se desarrollarán y el carácter cooperativo de las mismas.

En esta misma línea, Suárez $(2010,93)$ indica que, puesto que la relación cooperativa entre los miembros del grupo no "germina azarosamente", es responsabilidad del profesorado atender a algunos aspectos o dimensiones en el diseño de actividades de aprendizaje cooperativo para que estas sean exitosas. Estos aspectos están relacionados con la aclaración metas y tareas de equipo al inicio de la actividad, con la consolidación de grupos heterogéneos, con una distribución óptima de los recursos y el entorno educativo, con el desarrollo de habilidades cooperativas, con la distribución de roles y funciones intraequipo, y con la evaluación durante el proceso y el producto de la interdependencia.

Estos aspectos, comúnmente planteados y aceptados en la literatura, ponen de manifiesto la 
relevancia de ser considerados por el profesorado en cualquier actividad cooperativa que desarrolle. Pero, ¿'son aceptados también por los estudiantes? García, González y Mérida (2012), han desarrollado un estudio en el que se recogen una serie de dimensiones que vienen a indicar la importancia que tiene la visión de los estudiantes ante el aprendizaje cooperativo en aspectos relacionados con su concepción, funcionamiento y organización. Estas dimensiones son las que siguen:

- Concepción y significados que los estudiantes poseen acerca de la influencia del trabajo en grupo para su desarrollo cognitivo, social y académico.

- Utilidad del trabajo en grupo para su formación; más específicamente, para potenciar las interacciones sociales, el aprendizaje autónomo y el futuro desempeño profesional.

- Planificación del trabajo de los grupos por parte del profesorado; es decir, la cantidad, complejidad, coordinación y tutorización de los trabajos cooperativos.

- Criterios para organizar los grupos (criterios, composición, estabilidad temporal de los mismos).

- Normas de los grupos: esto es, la regulación de normas explicitas que arbitren el funcionamiento del grupo.

- Funcionamiento interno de los grupos (acciones realizadas durante el proceso de trabajo cooperativo).

- Eficacia del trabajo grupal (requisitos que favorecen mejores niveles de rendimiento y producción).

Las dimensiones planteadas por estos autores, dirigidas a valorar las actitudes de los estudiantes ante la organización y el funcionamiento del trabajo en grupo, permiten evaluar el proceso de aprendizaje cooperativo que se desarrolla en escenarios formativos universitarios, y proporciona, asimismo, la posibilidad de mejorar la actuación docente en el rediseño de estas acciones formativas. Esta es la finalidad última del trabajo que se presenta: conocer las valoraciones de los estudiantes respecto al trabajo en grupo en una experiencia de Aprendizaje Basado en Problemas.

$\mathrm{Si}$, como se ha señalado anteriormente, el diseño de acciones formativas basadas en el aprendizaje cooperativo requiere de la consideración de ciertas condiciones (ampliamente estudiadas), relacionadas con la interacción del grupo y con el tipo de agrupamiento (criterios, funcionamiento, normas, etc.), este trabajo se centra en las segundas condiciones, persiguiendo conocer las valoraciones de los estudiantes sobre la organización y el funcionamiento de los grupos de trabajo más idóneos para alcanzar un aprendizaje realmente cooperativo.

Cabe destacar, como indican (García, González y Mérida, 2012) que existe un porcentaje muy bajo de estudios que abordan el aprendizaje cooperativo en educación superior. Por ello, y porque, como se ha dicho anteriormente, el aprendizaje cooperativo en la universidad se considera una competencia básica que servirá para trasvasarla posteriormente a la profesión docente, creemos pertinente y de interés indagar más profundamente en este nivel educativo. Para el alcance del objetivo general que nos hemos planteado se proponen los siguientes objetivos específicos: primero, identificar las percepciones de los estudiantes sobre la planificación del trabajo en grupo por parte del profesorado en una experiencia de aprendizaje cooperativo; segundo, indagar en las valoraciones que los propios estudiantes realizan sobre el funcionamiento de los grupos de trabajo a los que han pertenecido en dicha experiencia; y tercero, conocer los criterios y las normas que los estudiantes consideran más adecuadas en la constitución de agrupamientos, así como los requisitos a los que atribuyen la eficacia del grupo.

\section{Método}

\subsection{Diseño}

La investigación se ha realizado utilizando una metodología cuantitativa, de corte exploratorio y descriptivo. Concretamente, el objetivo fundamental del estudio fue conocer y comprender lo que 
concierne a las valoraciones de los estudiantes sobre la organización y el funcionamiento de los agrupamientos en Educación Superior en una experiencia de aprendizaje concreta, realizada bajo la estrategia metodológica ABP.

\subsection{Muestra}

Para la elección de la muestra se llevoó a cabo un muestreo no probabilístico de muestras homogéneas (Hernández, Fernández y Baptista, 2010). La muestra estuvo constituida 75 por estudiantes pertenecientes a dos facultades o centros: Facultad de Educación de la Universidad de Murcia e ISEN, Centro Universitario adscrito a la Universidad de Murcia. Se eligió esta muestra dado que se corresponde con los estudiantes que han cursado materias en las que se ha aplicado la estrategia metodológica ABP (bajo el Proyecto de Innovación Docente "El ABP como estrategia metodológica para favorecer aprendizajes profundos y relevantes en futuros maestros y pedagogos"), que se basaba en la constitución de grupos cooperativos para la resolución de problemas. Dichas estrategias fueron utilizadas en la materia "La profesión docente en Educación Infantil" que es impartida en ambos centros por el mismo profesorado.

Tabla 1

Distribución de participantes por centro

\begin{tabular}{|c|c|c|c|c|c|}
\hline & & Frecuencia & Porcentaje & Porcentaje válido & $\begin{array}{l}\text { Porcentaje } \\
\text { acumulado }\end{array}$ \\
\hline \multirow{3}{*}{ Válidos } & Facultad de Educación & 40 & 53,3 & 53,3 & 53,3 \\
\hline & ISEN, Centro Univ. & 35 & 46,7 & 46,7 & 100,0 \\
\hline & Total & 75 & 100,0 & 100,0 & \\
\hline
\end{tabular}

Fuente: elaboración propia.

Como se puede apreciar en la tabla 1, de los 75 estudiantes que conformaron la muestra, 40 cursaban el grado de Educación Infantil en la Facultad de Educación y 35 cursaban ese mismo grado en ISEN.

Tabla 2

Distribución de participantes por género y centro

\begin{tabular}{clllll} 
& & & & \multicolumn{2}{c}{$\begin{array}{c}\text { Porcentaje } \\
\text { Válidos }\end{array}$} \\
\cline { 2 - 6 } & Mujer & 71 & 94,7 & 94,7 & 94,7 \\
\cline { 2 - 6 } & Hombre & 4 & 5,3 & 5,3 & 100,0 \\
\cline { 2 - 6 } & Total & 75 & 100,0 & 100,0 & \\
\hline
\end{tabular}

Fuente: elaboración propia.

Finalmente, y como se muestra en la tabla 2, del total de participantes del estudio, 71 fueron mujeres $(94.7 \%)$ y 4 hombres $(5,3 \%)$. Esta representación mayor de mujeres es lógica si tenemos en consideración que las titulaciones de Educación se configuran por una mayor presencia de mujeres entre sus estudiantes.

\subsection{Instrumento}

Para el estudio de las valoraciones de los estudiantes sobre la organización y el funcionamiento de los grupos de trabajo se utilizó el Cuestionario para el Análisis de la Cooperación en Educación Superior (Escala ACOES) (García Cabrera, González López y Mérida Serrano, 2012). Este instrumento recoge información de los estudiantes acerca de la importancia del trabajo cooperativo en su formación como futuro docente y sus percepciones acerca de la estructura y el funcionamiento de los equipos de trabajo en la metodología de aprendizaje cooperativo.

Se trata de una escala tipo Likert de cinco alternativas de respuesta de tal manera que 1 equivale a «totalmente en desacuerdo»y «totalmente de acuerdo». La escala ACOES presenta 49 ítems estructurados en siete bloques: a. Concepción del trabajo en grupo; b. Utilidad del trabajo en grupo para su formación; c. Planificación del trabajo de los grupos por parte del profesorado; d. Criterios 
para organizar los grupos; e. Normas de los grupos; f. Funcionamiento interno de los grupos; g. Eficacia del trabajo grupal. Al finalizar el cuestionario se incorporan tres cuestiones de respuesta abierta para que el alumnado pueda señalar las fortalezas, debilidades y propuestas para mejorar el trabajo grupal.

Para este estudio, concretamente, se analizarán los resultados extraídos de los ítems relativos a los bloques c, d, e, f y g. Es decir, las valoraciones de los estudiantes sobre la organización y el funcionamiento de los agrupamientos, dejando para un posterior análisis los bloques a y b y las cuestiones abiertas.

Por último, señalar que el análisis de la fiabilidad del instrumento presentó un coeficiente alfa de Cronbach de .840 .

\subsection{Procedimiento}

La aplicación del cuestionario se realizó dentro del aula y a través de la herramienta de cuestionarios de Google, donde se alojó previamente el cuestionario ACOES. Previo a su realización, se comentaron las instrucciones para su cumplimentación y los propósitos del estudio. Posteriormente, se procedió a la codificación de las respuestas en una base de datos para su tratamiento estadístico. Los análisis fueron realizados a través del paquete estadístico SPSS, versión 19 para Mac, utilizando la estadística descriptiva mediante el análisis de medias y frecuencias.

\section{Resultados}

En el presente apartado se presentan los resultados obtenidos tras la aplicación del cuestionario ACOES, atendiendo a cada uno de los bloques seleccionados y analizados para este estudio.

\subsection{Planificación del trabajo de los grupos por parte del profesorado}

En relación con el objetivo específico 1, "identificar las percepciones de los estudiantes sobre la planificación del trabajo en grupo por parte del profesorado en una experiencia de aprendizaje cooperativo", los resultados referidos a cantidad de trabajos solicitados muestran que la mayoría de los estudiantes los considera bastante adecuados (32\%) (véase Figura 1). 
Figura 1.

Porcentajes de acuerdo y desacuerdo en relación con el bloque Planificación

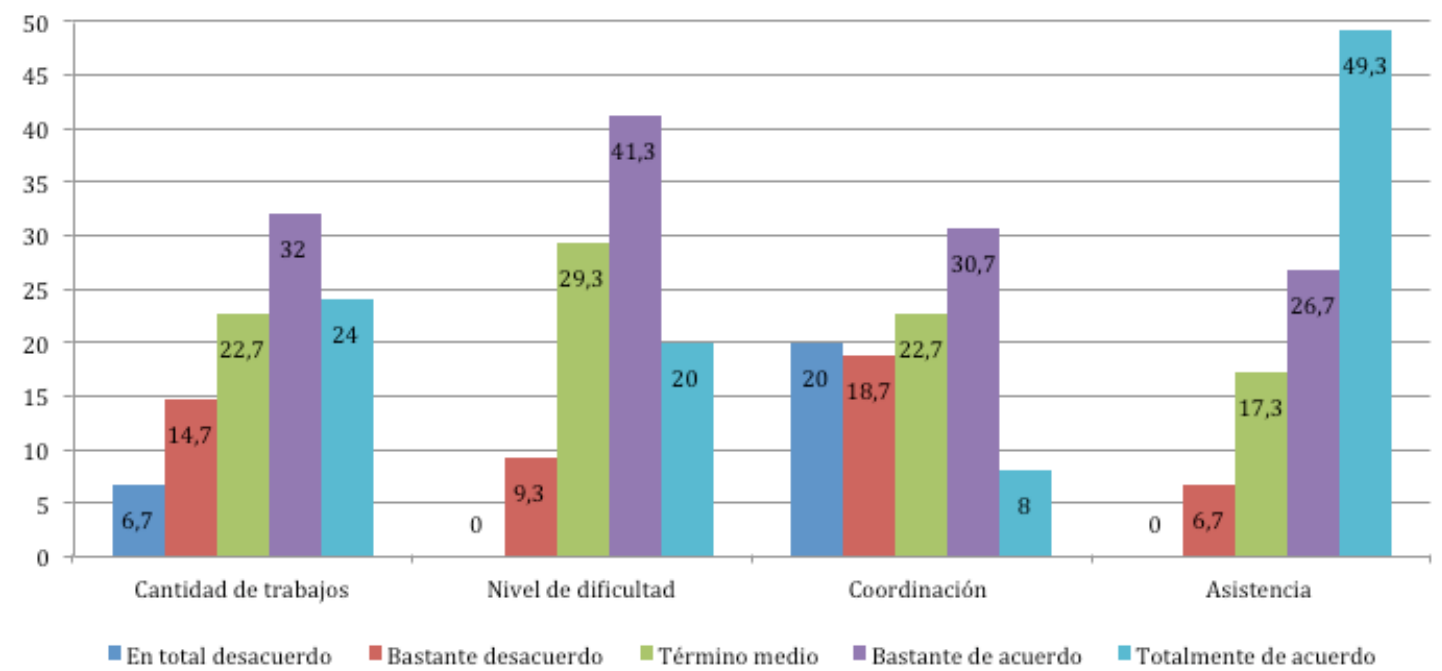

Fuente: elaboración propia.

Consideran, asimismo, que el nivel de dificultad de los trabajos es bastante adecuado para su formación (41,3\%). Casi la mitad de los estudiantes están totalmente de acuerdo en considerar que la asistencia a clases prácticas resuelve las dudas que surgen en la elaboración del trabajo en grupo y un $26,7 \%$ está bastante de acuerdo. Sin embargo, los estudiantes valoran que existe escasa coordinación entre los trabajos solicitados en las distintas asignaturas que cursan.

\subsection{Funcionamiento interno de los grupos}

Con el segundo objetivo específico se ha pretendido indagar en las valoraciones que los propios estudiantes realizan sobre el funcionamiento de los grupos de trabajo a los que han pertenecido en dicha experiencia.

En relación con el funcionamiento interno del grupo durante la experiencia de ABP (véase figura 2), encontramos grados de acuerdo altos en todos los ítems planteados. Están bastante de acuerdo $(37,3 \%)$ y totalmente de acuerdo $(34,7 \%)$ en que realizan una reunión inicial para planificar el trabajo en grupo. La mayoría de estudiantes indica que consultan la documentación básica aportada por la profesora $(53,3 \%)$ y también buscan información de otras fuentes $(53,3 \%)$. Consideran que toman decisiones de manera consensuada para garantizar la coherencia del trabajo $(42,7 \%)$ y que realizan puestas en común para que todos los miembros del grupo conozcan lo que cada uno de ellos hace.

Sin embargo, en relación con la participación equitativa de todos los miembros del grupo, se encuentran porcentajes de acuerdo dispares. No obstante, un porcentaje mayor de estudiantes están bastante de acuerdo $(25,3 \%)$ y totalmente de acuerdo $(25,3 \%)$ con que existe una participación equitativa. Respecto a la evaluación, encontramos porcentajes similares que indican que los grupos evalúan el trabajo que realizan y realizan propuestas de mejora 
Figura 2

Porcentajes de acuerdo y desacuerdo en relación con el bloque Funcionamiento interno del grupo

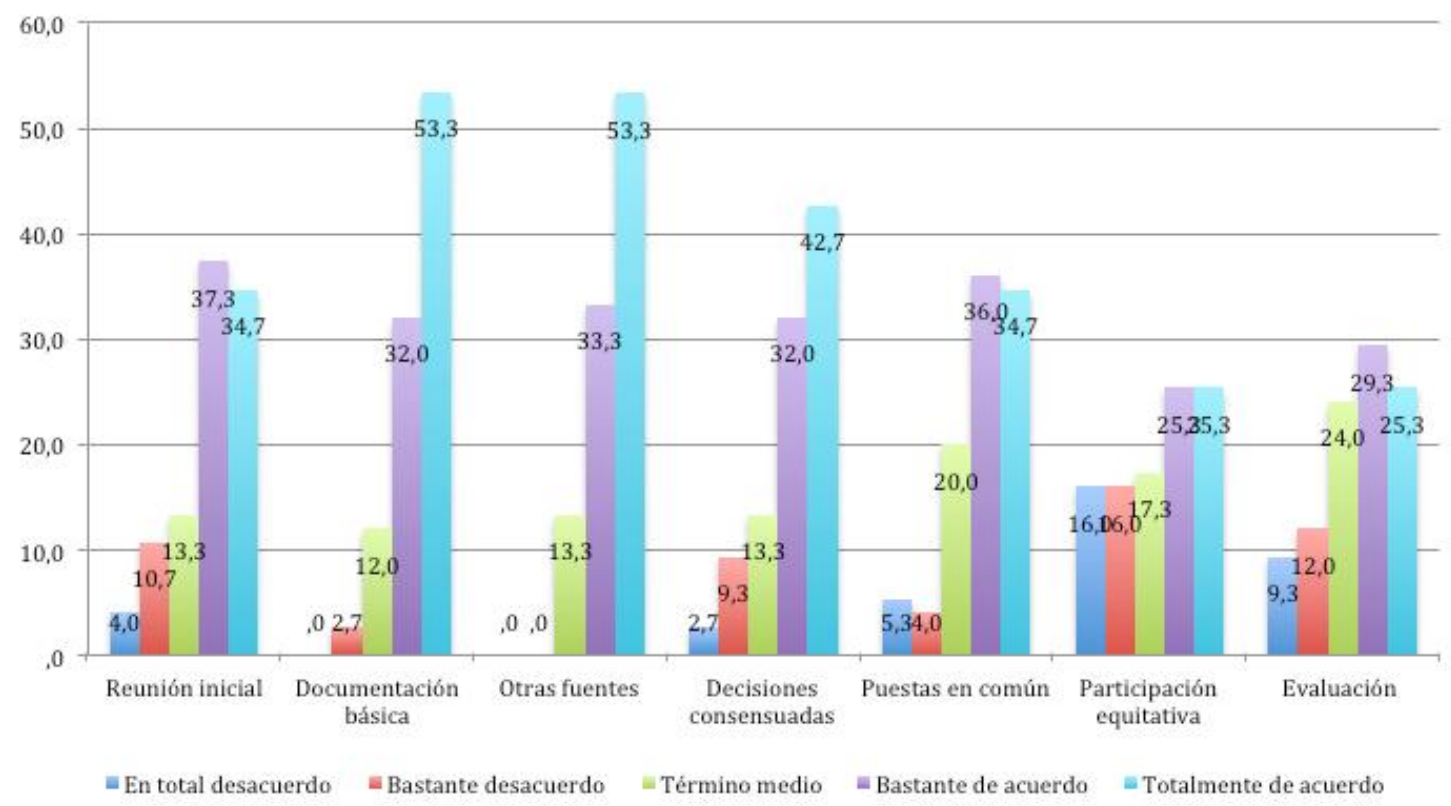

Fuente: elaboración propia.

\subsection{Criterios y normas para organizar los grupos y eficacia del trabajo grupal}

En relación al tercer objetivo específico, por un lado, los resultados de este estudio han permitido conocer los criterios y las normas que los estudiantes consideran más adecuadas en la constitución de agrupamientos; y por otro lado, los requisitos a los que atribuyen la eficacia del grupo.

La siguiente figura muestra las concepciones que los estudiantes poseen respecto de los criterios que se han de tener en cuenta para organizar los grupos. Concretamente, un alto porcentaje de estudiantes considera que los agrupamientos pueden realizaros ellos mismos teniendo en cuenta criterios de amistad, pero ese porcentaje es superado por los estudiantes que están bastante de acuerdo $(33,3 \%)$ o muy de acuerdo $(32 \%)$ en considerar que deben realizarlos ellos mismos atendiendo a criterios académicos. Existe, sin embargo, cierto desacuerdo en considerar que dichos agrupamientos han de realizarse por parte del profesorado teniendo en cuenta criterios académicos, con un porcentaje del $22,7 \%$ que manifiesta su total desacuerdo y un $13,3 \%$ que está bastante desacuerdo. 
Figura 3

Porcentajes de acuerdo y desacuerdo en relación al bloque Criterios para organizar los grupos

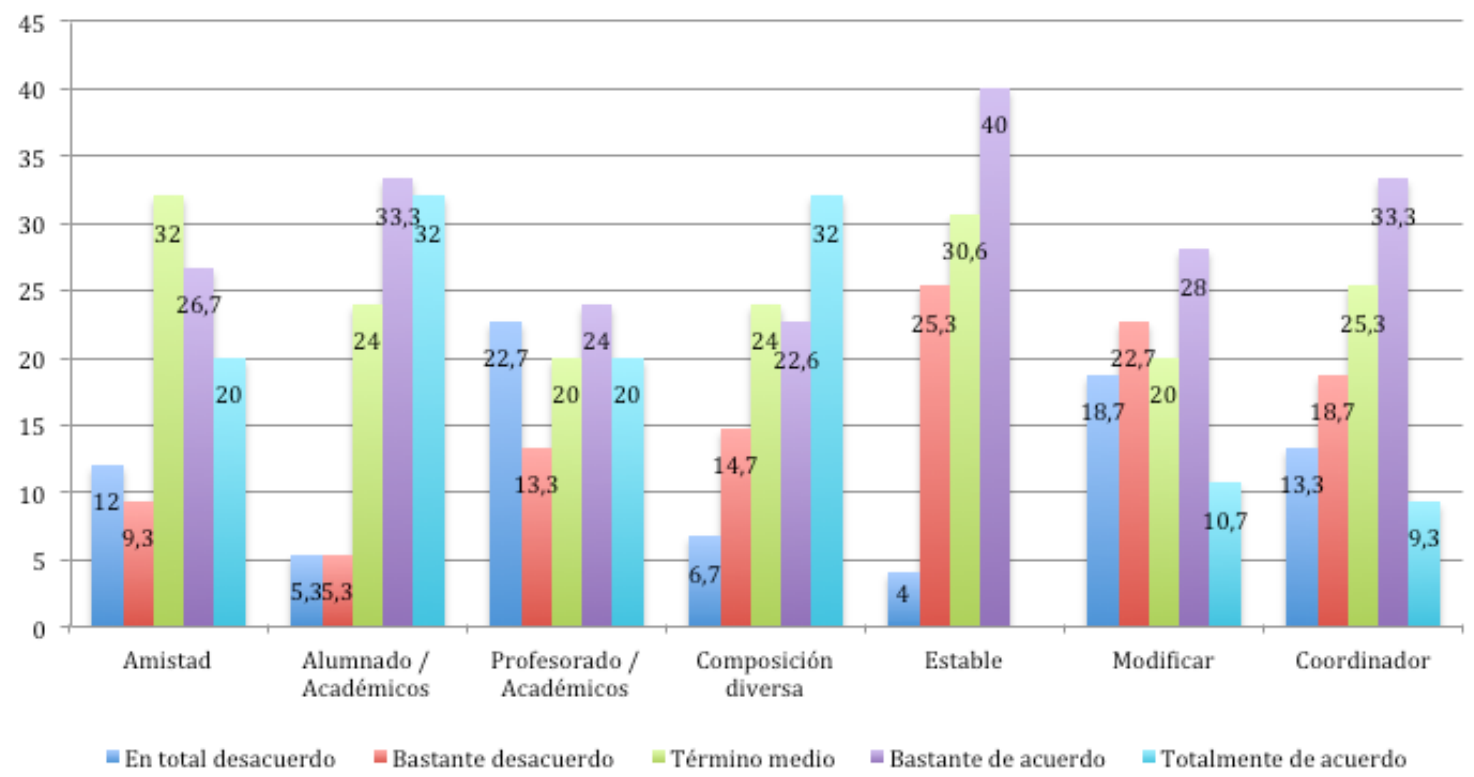

Fuente: elaboración propia.

Respecto a la composición de los grupos, la mayoría de estudiantes (32\%) está completamente de acuerdo en la idea de que los grupos han de poseer una composición diversa en cuanto a edad, sexo, experiencias, etc. De igual modo, se aprecia que el $40 \%$ de los estudiantes concibe que el grupo debe permanecer estable a lo largo de la asignatura. Estos porcentajes se dispersan cuando se les pregunta si los grupos deben modificarse en la realización de tareas de una misma asignatura, hallando porcentajes dispares pero que descienden al 10,7\% de estudiantes que está de acuerdo. Por último, respecto a la incorporación de un coordinador o coordinadora de grupo, el 33,3\% de los estudiantes está de acuerdo con que se designe.

En relación con el número de estudiantes por grupo, tal y como se muestra en la Tabla 3, los estudiantes consideran que un grupo debe tener una media de 3,17 participantes, es decir, entre 3 y 4 estudiantes.

Tabla 3

Número de estudiantes por grupo

\begin{tabular}{ccccc}
\hline $\mathrm{N}$ & Mínimo & Máximo & Media & Desv. Típ. \\
\hline 75 &, 00 & 7,00 & 3,17 & 1,77
\end{tabular}

Fuente: elaboración propia.

Otro aspecto analizado han sido las normas de funcionamiento de un grupo (véase Figura 4). Respecto a esta cuestión, los estudiantes han manifestado un amplio desacuerdo en considerar que no deben existir normas (54,7\%). Por el contrario, se considera que las normas deben existir: un elevado porcentaje está bastante de acuerdo en que estas deben ser establecidas por el alumno (36\%) y un porcentaje ligeramente menor está completamente de acuerdo con esta afirmación (21,3\%). Estos porcentajes disminuyen cuando se les plantea la posibilidad de que las normas sean establecidas únicamente por el profesorado. A pesar de ello, existe un acuerdo generalizado en considerar que las normas deben ser negociadas entre profesorado y alumnado, estando totalmente de acuerdo el 42,7\% y bastante de acuerdo el 29,3\% de los estudiantes. Asimismo, se considera que estas normas deben estar recogidas en un documento $(34,7 \%)$. 
Figura 4

Porcentajes de acuerdo y desacuerdo en relación con el bloque Normas de los grupos



Fuente: elaboración propia.

En relación con los roles que se van a desempeñar en el grupo, no existe consenso en suponer su necesidad, el 29,3\% de los estudiantes no se posiciona. Sin embargo, sí que existe un elevado porcentaje de estudiantes que está de acuerdo en la necesidad de incluir las consecuencias que tendrían para los participantes el hecho de no cumplir con sus responsabilidades.

Los estudiantes están de bastante de acuerdo $(30,7 \%)$ en que se debe concretar el horario y lugar de las reuniones. Igualmente, la asistencia a las reuniones también es un aspecto importante para los estudiantes, pues en su mayoría (el 40\% totalmente de acuerdo y el 30,7\% bastante de acuerdo) consideran que entre las normas del grupo se debe de incluir la obligatoriedad de asistir a las mismas.

Finalmente, para conocer los requisitos a los que atribuyen la eficacia del grupo (véase Figura 5), se les ha preguntado si el rendimiento del grupo mejora si el profesorado facilita pautas claras respecto de las actividades a desarrollar. En este aspecto, más de la mitad de los estudiantes están de acuerdo con esta idea. Estos porcentajes son muy similares en los todos los ítems planteados, donde los porcentajes de estudiantes que sitúan su posicionamiento en "bastante de acuerdo" y "totalmente de acuerdo" superan, en gran medida, la mitad de los participantes.

Estos resultados evidencian la importancia que los estudiantes otorgan a las actuaciones del docente en el trabajo en grupo. Se perciben porcentajes de acuerdo mayores en el establecimiento de unas pautas claras por parte del docente $(53,3 \%)$ así como en el control de la asistencia $(50,7 \%)$. 
Figura 5

Porcentajes de acuerdo y desacuerdo en relación con el bloque Eficacia del grupo

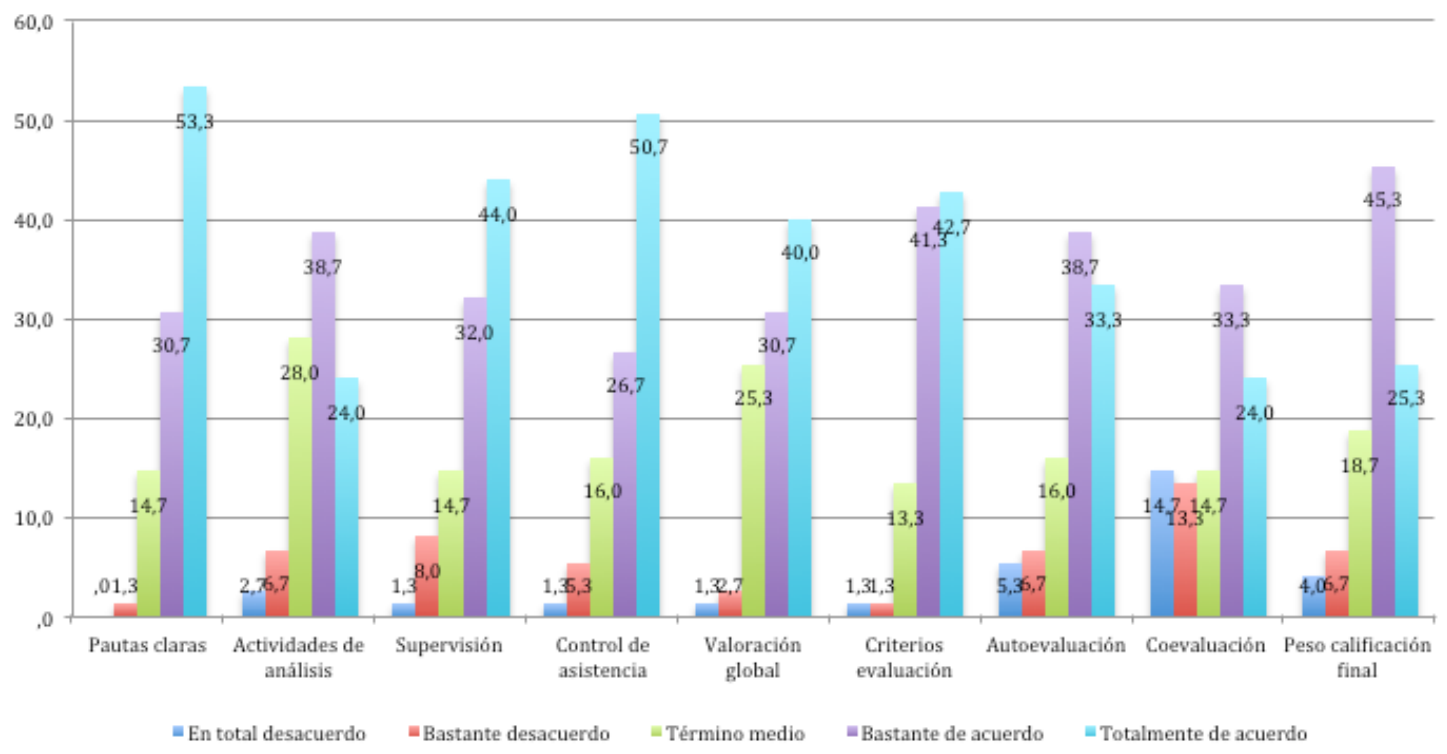

Fuente: elaboración propia.

\section{Discusión y conclusiones}

El objetivo fundamental de este trabajo fue conocer las valoraciones de los estudiantes sobre la organización y el funcionamiento de los grupos de trabajo más idóneos para alcanzar un aprendizaje realmente cooperativo, así como analizar sus percepciones sobre la experiencia cooperativa de aprendizaje en la que habían participado.

La valoración realizada por los estudiantes de la experiencia de aprendizaje cooperativo en la que participaron puso de manifiesto el desarrollo de aspectos o dimensiones que Suárez (2010) califica como exitosos en las actividades de aprendizaje cooperativo. Estos aspectos, relacionados con la actuación del docente en el diseño de actividades, mostraron el acuerdo existente en considerar que la cantidad de trabajos y el nivel de exigencia eran adecuados, y se valoró positivamente la asistencia a clase como lugar para la resolución de dudas y problemas. Sin embargo, hubo menor acuerdo en considerar la existencia de coordinación entre los trabajos de las distintas asignaturas. Este último aspecto coincide con la investigación realizada por García Cabrera (2011). En este sentido, los estudiantes consideraron la coordinación docente como un aspecto susceptible de mejora para que exista mayor conexión entre los procesos de aprendizaje cooperativos y las actividades que se solicitan.

Respecto al funcionamiento interno del grupo en la experiencia de aprendizaje, los estudiantes mostraron un conocimiento exhaustivo del modo en que han de proceder para realizar un aprendizaje realmente cooperativo y manifestaron que lo llevaron a la práctica en la experiencia de aprendizaje cooperativo para la resolución de problemas ABP. Concretamente, se encontraron grados de acuerdo muy elevados respecto al funcionamiento del grupo: reuniones iniciales, diversas fuentes consultadas para la realización de los trabajos, puestas en común de forma periódica, toma de decisiones consensuada, etc. Sería conveniente incidir en este aspecto para corroborar si dichas valoraciones fueron, efectivamente, trasladadas a la práctica.

En relación con los criterios y normas para organizar los grupos, los estudiantes coincidieron con los planteamientos desarrollados en el marco teórico de este trabajo (Ovejero, 1990; Lobato, 1998; García, Traver y Candela, 2001) al señalar que la configuración de los grupos ha de ser heterogénea y agrupar entre tres y cinco estudiantes. Sin embargo, consideraron que las agrupaciones se deben realizar a criterio de los estudiantes (en contraposición a Colomina y Onrubia, 2004) en función, principalmente, de criterios académicos. Asimismo, se valoró positivamente la duración estable en 
el tiempo de los grupos, criterio que Pujolás (2008) considera imprescindible para que se desarrolle un aprendizaje cooperativo auténtico.

Hubo también un acuerdo generalizado en considerar que cualquier grupo de trabajo ha de configurarse bajo unas normas, que dichas normas tendrían que ser consensuadas entre profesorado y alumnado, y ser reflejadas en un documento para su cumplimiento. Entre dichas normas consideraron prioritario incidir en la obligatoriedad de la asistencia a las sesiones grupales. La definición de roles y funciones, sin embargo, no estuvo tan bien valorada, aun siendo un requisito imprescindible para el reparto de responsabilidades en el grupo (Ovejero, 1990; Lobato, 1998; García, Traver y Candela, 2001).

Finalmente, y en cuanto a los requisitos que permiten la eficacia del grupo, los estudiantes valoraron que el rendimiento del grupo mejora si las actividades planteadas por el profesorado requieren de análisis, reflexión, debate y crítica; si el profesorado controla la asistencia regular a clase y supervisa el trabajo en grupo; si controla la asistencia regular a clase; si los trabajos se valoran adecuadamente en la calificación global de la asignatura; si el profesorado informa previamente sobre los criterios de evaluación; y si los estudiantes se autoevalúan y evalúan a sus compañeros. Se señalaron, por tanto, las condiciones que, según Kagan (1999) y Atxurra, VillardónGallego y Calvete (2015), el profesorado ha de diseñar y estructurar para que la situación de enseñanza y aprendizaje sea realmente cooperativa.

Estos resultados han permitido conocer cuál es la perspectiva del alumnado respecto al funcionamiento real de los grupos de trabajo en una experiencia de aprendizaje ABP. Por ello, las evidencias planteadas deben tomarse con cautela pues muestran un diagnóstico de la realidad de las agrupaciones vista, solamente, desde la perspectiva de los estudiantes.

A modo de síntesis, podemos concluir que esta experiencia de aprendizaje cooperativo, aun con las dificultades y limitaciones que haya podido presentar, ha iniciado a los estudiantes en el aprendizaje de habilidades básicas para la cooperación. Efectivamente, como bien indican Fombona, Iglesias y Lozano (2016), dicho aprendizaje habrá de ser consolidado en base diversas experiencias formativas y didácticas que tengan lugar en la Universidad, para poder transvasarlas posteriormente a los escenarios profesionales.

\section{Referencias}

ANECA (2016). Evaluación sobre la propuesta de modificación de plan de estudios. Recuperado de: http://www.um.es/documents/299436/3179530/Notificacion_INFORME+FAVORABLE.pd f/85207a1e-9e54-49da-a1d7-345fae6bda4d.

Atxurra, C., Villardón-Gallego, L., \& Calvete, E. (2015). Diseño y validación de la Escala de Aplicación del Aprendizaje Cooperativo (CLAS). Revista de Psicodidáctica, 20(2), pp. 339-357. doi: 10.1387/RevPsicodidact.11917.

Bolívar, A. (2013). La lógica del compromiso del profesorado y la responsabilidad de la escuela. Una nueva mirada. Revista Iberoamericana sobre Calidad, Eficacia y Cambio en Educación, 11 (2), pp. 6186.

Colomina, R., \& Onrubia, J. (2004). Interacción educativa y aprendizaje escolar: la interacción entre alumnos. En Coll, C., Palacios, J., \& Marchesi, A. (Comps.), Desarrollo Psicológico y Educación (pp. 415-436). Madrid: Alianza Editorial.

Darling-Hammond, L. (2008). The case for university-based teacher education. En Cochran, M.S., Feiman-Nemser, S., Mcintyre, D.J., \& Demers, K.E. (Eds.), Handbook of Research on Teacher Education. Enduring Questions in Changing Contexts (pp. 333-346). New York: Routledge.

Escudero, J.M. (2009). Comunidades docentes de aprendizaje, formación del profesorado y mejora de la educación. Agora para la EFy el Deporte, 10, pp. 7-31.

Fombona, J., Iglesias, M.J., \& Lozano, I. (2016). El trabajo colaborativo en la educación superior: una competencia profesional para los futuros docentes. Educação \& Sociedade, 37(135), pp. 519538. doi: 10.1590/ES0101-73302016147914.

García Cabrera, M.M. (2011). Análisis del trabajo en grupo como estrategia formativa en las titulaciones de 
ciencias de la educación de la universidad de córdoba (Tesis inédita de Doctorado). Universidad de Córdoba, España.

García, M.M., González, I., \& Mérida, R. (2012). Validación del cuestionario de evaluación ACOES. Análisis del trabajo cooperativo en Educación Superior. Revista de Investigación Educativa, 30(1), pp. 87-109.

García, R., Traver, J., \& Candela, I. (2001). Aprendizaje cooperativo. Fundamentos, características y técnicas. Madrid: CCS.

Goikoetxea, E., \& Pascual, G. (2002). Aprendizaje cooperativo: bases teóricas y hallazgos empíricos que explican su eficacia. Revista Educación XXI, 5, pp. 227-247. doi: 10.5944/educxx1.5.1.392.

González, N., \& García, M.R. (2007). Aprendizaje Cooperativo como estrategia de EnseñanzaAprendizaje en Psicopedagogía (UC): repercusiones y valoraciones de los estudiantes. Revista Iberoamericana de Educación, 42(6), pp. 1-13.

Hancock, D. (2004). Cooperative learning and peer orientation effects on motivation and achievement. Journal of Educational Research, 97(3), pp. 159-161. doi: 10.3200/JOER.97.3.159-168.

Hernández, R.; Fernández Collado, C., \& Baptista Lucio, P. (2010). Metodología de la investigación (5 ed.). México: McGRAW-HILL.

Johnson, D. W., \& Johnson, R. T. (1987). A meta-analysis of cooperative, competitive and individualistic goal structures. Hillsdale: Lawrence Erlbaum Associates.

Johnson, D. W., Johnson, R. T., \& Holubec, E. J. (1999). El aprendiraje cooperativo en el aula. Buenos Aires: Paidós.

Jourand, C., Adé, D., Sève, C., Komar, J., \& Thouvarecq, R. (2017). Dynamics of student interactions: an empirical study of orienteering lessons in physical education. Physical Education and Sport Pedagogy, 23(2). doi: 10.1080/17408989.2017.1342790.

Kagan, S. (1999). Cooperative Learning. San Clemente: Resources for Teachers, Inc.

Lledó, A., \& Perandones, T.M. (2011). Hacia una cultura colaborativa entre el profesorado universitario. Revisión y fundamentación teórica del trabajo colaborativo. En Gómez, M.C., \& Álvarez, J.D. (Coords.), El trabajo colaborativo como indicador de calidad del Espacio Europeo de Educación Superior (pp. 125-138). Alcoy: Editorial Marfil.

Lobato, C. (1998). El trabajo en grupo. Aprendizaje cooperativo en Secundaria. Bilbao: Servicio de Publicaciones Universidad del País Vasco.

Martínez, P., \& González, N. (2018). Las competencias transversales en la universidad: propiedades psicométricas de un cuestionario. Educación XX1 (en prensa). doi. 10.5944/educXX1.15662.

Michavila, F., Martínez, J.M., Martín-González, M., García-Peñalvo, F.J., \& Cruz-Benito, J. (2016). Barómetro de empleabilidad y empleo de los universitarios en España, 2015 (Primer informe de resultados). Madrid: Observatorio de Empleabilidad y Empleo Universitario.

Miró, J. (2010). Para qué sirven las competencias transversales. Recuperado de: http://bioinfo.uib.es/ joemiro/CTens/PorqueCT.pdf.

Ovejero, A. (1990). El aprendizaje cooperativo, una alternativa eficaz a la enseñanza tradicional. Barcelona: PPU.

Pérez, A.M. (2008). Efectos del aprendizaje cooperativo en la adaptación escolar. Revista de Investigación Educativa, 26(1), pp. 73-94.

Prieto, L. (2007). El aprendizaje cooperativo. Madrid: PPC.

Pujolás, P. (2008). El aprendizaje cooperativo como recurso y como contenido. Revista Aula de Innovación Educativa, 170. Recuperado de: http://www.grao.com/revistas/aula/170competencias-en-ciencias-sociales/el-aprendizaje-cooperativo-como-recurso-y-como-contenido.

Pujolás, P. (2009). La calidad de los equipos de aprendizaje cooperativo. Algunas consideraciones para el cálculo del grado de cooperatividad. Revista de Educación, 349, pp. 225-239.

Real Decreto 1027/2011, de 15 de julio, por el que se establece el Marco Español de Cualificaciones para la Educación Superior (MECES). (BOE de 3 de agosto).

Real Decreto 1393/2007, de 29 de octubre, por el que se establece la ordenación de las enseñanzas universitarias oficiales. (BOE de 30 de octubre).

Real Decreto 43/2015, de 2 de febrero, por el que se modifica el Real Decreto 1393/2007, de 29 de octubre, por el que se establece la ordenación de las enseñanzas universitarias oficiales, y el Real Decreto 99/2011, de 28 de enero, por el que se regulan las enseñanzas oficiales de doctorado. (BOE de 3 de febrero). 
Real Decreto 861/2010, de 2 de julio, por el que se modifica el Real Decreto 1393/2007, de 29 de octubre, por el que se establece la ordenación de las enseñanzas universitarias oficiales. (BOE de 3 de Julio).

Real Decreto 96/2014, de 14 de febrero, por el que se modifican los Reales Decretos 1027/2011, de 15 de julio, por el que se establece el Marco Español de Cualificaciones para la Educación Superior (MECES), y 1393/2007, de 29 de octubre, por el que se establece la ordenación de las enseñanzas universitarias oficiales. (BOE de 5 de Marzo).

Rodríguez de Guzmán, J. (2012). Comunidades de aprendizaje y formación del profesorado. Tendencias Pedagógicas, 19, pp. 67-86. Recuperado de: https://revistas.uam.es/tendenciaspedagogicas/article/view/2002.

Serrano, J. M., Pons, R. M., \& González-Herrero, M. E. (2007). El aprendizaje cooperativo y la organización cooperativa de la actividad docente. México: Trillas.

Slavin, R. (1999). Aprendizaje cooperativo. Teoría, investigación y práctica. Argentina: Aique.

Suárez, C. (2010). Cooperación como condición social de aprendizaje. Barcelona: UOC. 
